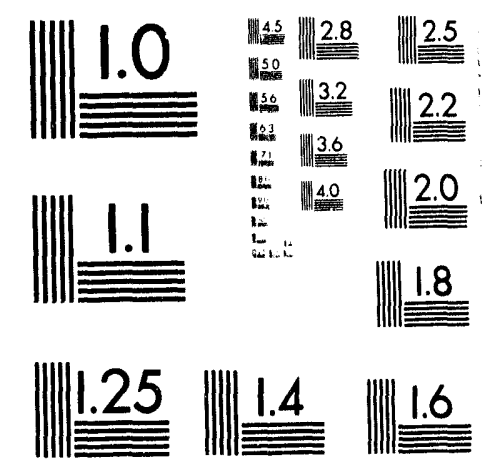



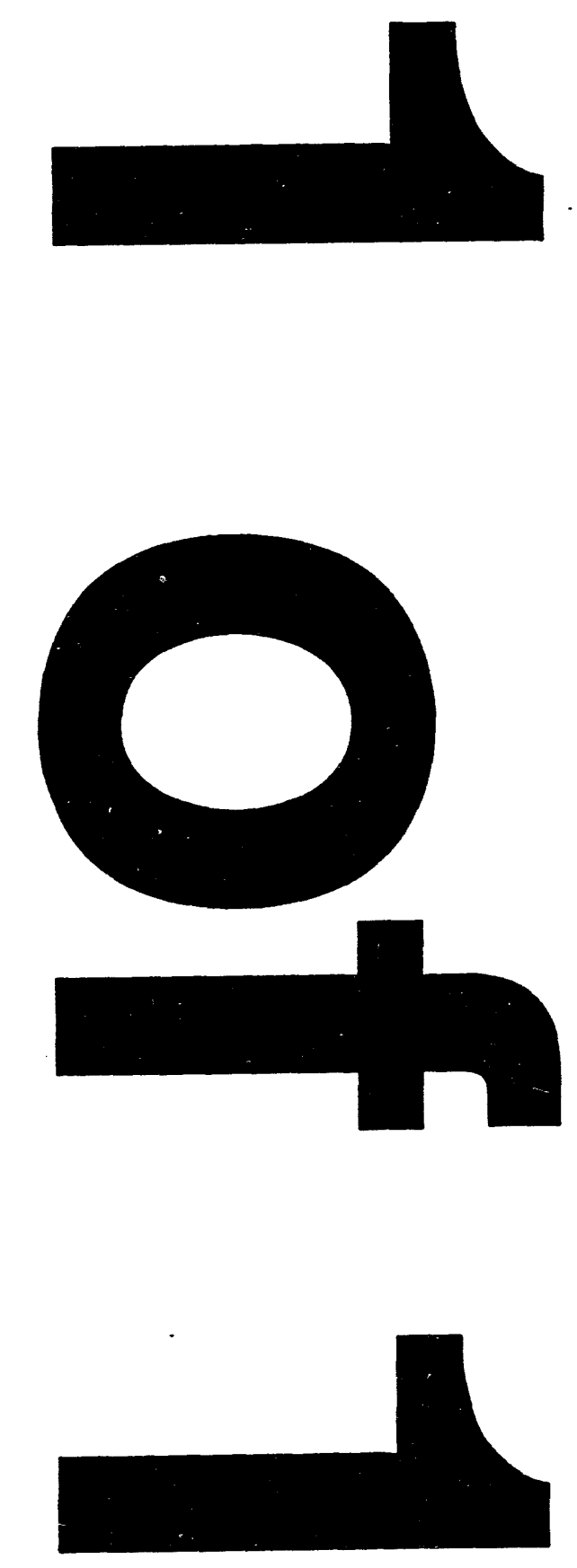


\title{
PROJECTILE TRANSVERSE MOTION AND STABILITY IN ELECTROMAGNETIC INDUCTION LAUNCHERS
}

\author{
I. R. Shokair \\ Conf $-940481-3$
}

Sandia National Laboratories

Beam, Plasma, and Electromagnetic Theory Department

P. O. Box 5800

Albuquerque, New Mexico 87185-1186

\begin{abstract}
The transverse motion of a projectile in an electromagnetic induction launcher is considered. The equations of motion for translation and rotation are derived assuming a rigid projectile and a flyway restoring force per unit length that is proportional to the local displacement. Linearized transverse forces and torques due to energized coils are derived for displaced or tilted armature elements based on a first order perturbation method. The resulting equations of motion for a rigid projectile composed of multiple elements in a multicoil launcher are analyzed as a coupled oscillator system of equations and a simple linear stability condition is derived. The equations of motion are incorporated into the 2-D Slingshot circuit code and numerical solutions for the transverse motion are obtained. For a launcher with a $10 \mathrm{~cm}$ bore radius with a $40 \mathrm{~cm}$ long solid armature, we find that stability is achieved with a restoring force (per unit length) constant of $k \approx 1 \times 10^{8}$ $\mathrm{N} / \mathrm{m}^{2}$. For $k=1.5 \times 10^{8} \mathrm{~N} / \mathrm{m}^{2}$ and sample coil misalignment modeled as a sine wave of $1 \mathrm{~mm}$ amplitude at wavelengths of one or two meters, the projectile displacement grows to a maximum of $4 \mathrm{~mm}$. This growth is due to resonance between the natural frequency of the projectile transverse motion and the coil displacement wavelength. This resonance does not persist because of the changing axial velocity. Random coil displacement is also found to cause roughly the same projectile displacement. For the maximum displacement a rough estimate of the transverse pressure is 50 bars. Results for a wound armature with uniform current density throughout show very similar displacments.
\end{abstract}

\section{Introduction}

The electromagnetic induction launcher accelerates a conducting armature by inducing armature currents opposite to the coil current, which result in a repulsive axial force that accelerates the armature ${ }^{(1-2)}$. Because of the finite resistivity the armature current decays and the magnetic field diffuses into the armature. For a 
solid armature, if the firing position of the coils is advanced (slipped) to account for field diffusion, near constant axial acceleration can be maintained ${ }^{(3)}$. For a wound armature ${ }^{(4)}$, no slipping is needed, but there is still field diffusion due to the finite resistivity resulting in an $L / R$ decay of the acceleration. Because of the favorable distribution of current density in a wound armature, voltage reversal can be used to significantly improve performance.

Although the acceleration is only in the axial direction, there are very large radial forces that act on both armature and coils. Slight mis-alignments of coils can result in net transverse forces and torques on the projectile resulting in transverse motion. It is very important to insure that this motion is stable and tolestimate the maximum amplitudes and induced stresses that result. This is the subject of this paper. In section II we derive the equations of motion for both translation and rotation assuming a rigid projectile. It is assumed that the flyway tube restoring forces per unit length are proportional to the local displacement. In sections III we derive the electromagnetic forces and torques that result when a armature element is displaced or tilted from the axis of a coil, based on a first order perturbation method. In section IV we write the equations of motion, for a multiple element projectile in a real launcher with many coils, in the form of coupled oscillators and consider the question of stability. Implementation of the transverse motion calculation in the Slingshot code ${ }^{(5)}$ is then described in section $\mathrm{V}$. We also show numerical results of transverse motion using the modified Slingshot for a 20 meter long launcher with a $20 \mathrm{~cm}$ bore for both solid and wound armatures. Finally in section VI, the results are summarized.

\section{Transverse Equations of Motion}

In the one dimensional case the projectile transverse motion can be described by two variables $x_{0}$, the displacement of the projectile center of mass and $\theta$, the angle of rotation of the projectile axis with respect to the unperturbed axis. This is illustrated in Fig. (1). The projectile is assumed to be rigid and symmetric with respect to the center of mass and is assumed to have no spin. The extension to spinning projectiles and two transverse dimensions will be briefly considered at the end of this section. The projectile displacement is given as a function of time and distance along the projectile by:

$x\left(t ; z^{\prime}\right)=x_{o}+z^{\prime} \sin \theta \quad \sim x_{o}+z^{\prime} \theta$

where $z^{\prime}$ is the length along the projectile measured from the center. We assume that the transverse forces due to the coils are given per unit length by $f_{c}\left(t ; z^{\prime}\right)$ and the flyway restoring force per unit length is given by: $f_{r}\left(t ; z^{\prime}\right)=-k x\left(t ; z^{\prime}\right)$. We can write the equation of motion for the center of mass by integrating over all the transverse forces and we get: 


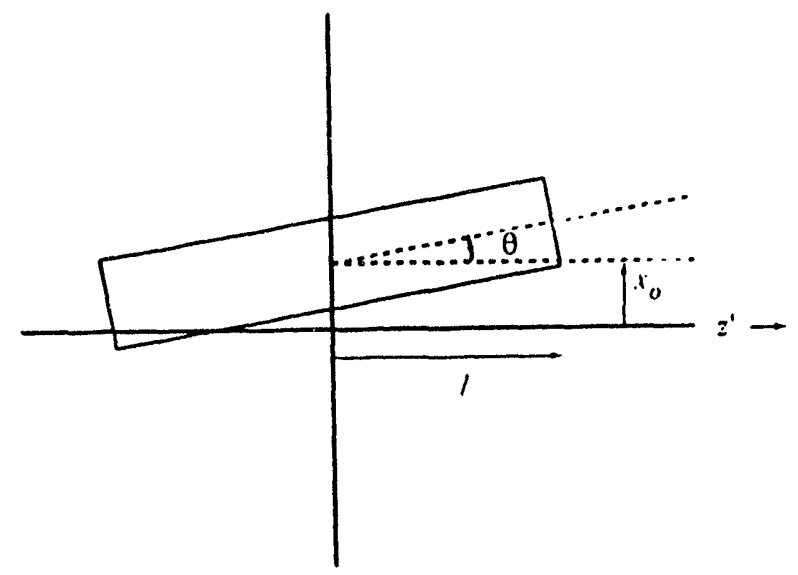

Fig. 1. Geometry of displaced projectile

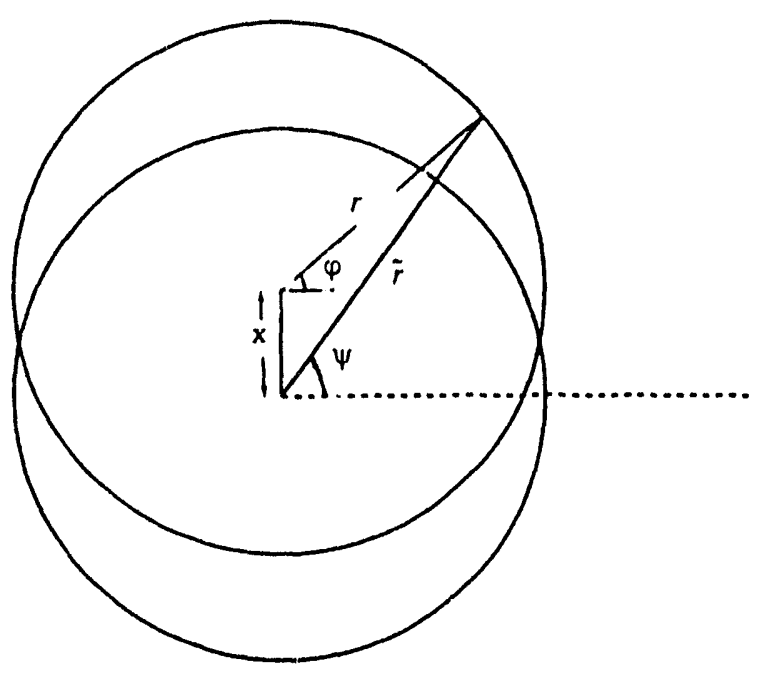

Fig. 2. Cross section of displaced projectile

where $M_{p}$ is the total projectile mass and $l$ is the half length. The equation for the rotation angle $\theta$ is more complex and can be derived by considering the projectile angular momentum. The angular momentum with respect to a fixed point (in the lab frame) located on the unperturbed axis is found to be:

$\vec{L}=M_{p}\left(-x_{o} v_{z}+\frac{d x_{o}}{d t} z+\frac{l^{2}}{3} \frac{d \theta}{d t}\right) \hat{y}$

where $v_{z}$ is the axial speed and $z$ is the distance of the projectile center of mass from the fixed reference point. The equation of motion is: $d \vec{L} / d t=\vec{\tau}$, where $\vec{\tau}$ is the applied torque. The resulting equation is:

$M_{p}\left(-x_{o} \frac{d v_{z}}{d t}+z \frac{d^{2} x_{o}}{d t^{2}}+\frac{l^{2}}{3} \frac{d^{2} \theta}{d t^{2}}\right)=\int_{-l}^{l} d z^{\prime}\left(z^{\prime}+z\right)\left(f_{c}+f_{r}\right)-\int d^{3} r^{\prime} \tilde{x} F_{z}$

where $F_{z}$ is the axial force per unit volume on the projectile, $\tilde{x}$ is the monient arm given by: $\tilde{x}=x_{o}+z^{\prime} \theta+r^{\prime} \sin \left(\varphi^{\prime}\right)$, and the integral over $d^{3} r^{\prime}$ is over the projectile volume, that is $d^{3} r^{\prime}=r^{\prime} d r^{\prime} d \varphi^{\prime} d z^{\prime}$ with $r^{\prime}$ and $\varphi^{\prime}$ measured with respect to the projectile axis. Since the axial equation of motion just gives the acceleration in terms of the total axial force, that is $M_{p} \frac{d v_{z}}{d t}=\int d^{3} r^{\prime} F_{z}$, and $d^{2} x_{0} / d t^{2}$ is described by Eq. (2), Eq. (4) reduces to: 
$\frac{M_{p}}{3} l^{2} \frac{d^{2} \theta}{d t^{2}}=\int_{-l}^{l} d z^{\prime} z^{\prime}\left[f_{c}\left(t ; z^{\prime}\right)-\theta(t) f_{z}\left(t ; z^{\prime}\right)\right]-\int d^{3} r^{\prime}\left[r^{\prime} \sin \varphi^{\prime}\right] F_{z}-\frac{2}{3} l^{3} k \theta(t)$

where $f_{z}$ is the axial force per unit length, that is $F_{z}$ integrated over the projectile cross section. The first term on the RHS of Eq. (5) is torque due to coil transverse forces and can be either stabilizing or destabilizing. The second term is the torque due to the axial force and is stabilizing if the force is concentrated toward the front of the projectile and destabilizing if it is concentrated toward the rear. The third term is the torque due to asymmetry of the axial force in the azimuthal coordinate. This also can be either stabilizing or destabilizing. The fourth term is the restoring torque due to reaction of the flyway tube and is always stabilizing. Thus if the coil-projectile interactions are known, the above equations can be solved and the flyway tube displacement and stress profiles can be obtained.

For the case of a projectile with spin, all three dimensions have to be considered. If the projectile spins with an angular frequency $\omega$, and the rotation angles along $\mathrm{x}$ and $\mathrm{y}$ are denoted by $\theta_{\mathrm{x}}$ and $\theta_{\mathrm{y}}$, then for small rotation angles, the angular momentum vector is given by: $\quad \vec{L}=I_{o} \omega\left(\hat{z}+\theta_{x} \hat{x}+\theta_{y} \hat{y}\right)$. where $\mathrm{I}_{0}$ is the moment of inertia given by: $I_{o}=M_{p} R^{2} / 2$. With the assumption of axi-symmetry (to zeroth order), the torque in the axial direction is zero and thus the axial component of $d \vec{L} / d t$ is zero, which implies that $\omega$ is constant in time. Thus we have: $\frac{d \vec{L}}{d t}=I_{o} \omega\left(\frac{d \theta_{x}}{d t} \hat{x}+\frac{d \theta_{y}}{d t} \hat{y}\right)$. The effect of these spin terms has to be compared with other terms in Eq. (5). For a fixed torque, and if $\omega$ is large, $d \theta / d t$ will be small and thus spin will have the effect of reducing rotational motion. We also note that since the spin term in the angular momentum equation is first order in $\mathrm{d} \theta / \mathrm{dt}$, it is a damping term. How much damping there is will depend on the relative magnitudes of the terms.

\section{Coil-Projectile Interactions}

We first consider the transverse force and torque on a armature element that results when it is displaced co-axially from a coil by a small amount. Using the fact that the magnetic flux at a armature element due to coil current $\mathrm{I}_{\mathrm{i}}$ is given by: $\Phi_{i j}=M_{i j} I_{i}$, where $\mathrm{M}_{\mathrm{ij}}$ is the mutual inductance between the coil (denoted by $\mathrm{i}$ ) and the armature element (denoted by $\mathrm{j}$ ), we find that the axial and radial magnetic fields can be written as:

$$
B_{z}(r, z)=\frac{I_{i}}{2 \pi r} \frac{\partial M_{i j}}{\partial r} \text {, and } B_{r}(r, z)=\frac{-I_{i}}{2 \pi r} \frac{\partial M_{i j}}{\partial z}
$$

Now if the armature element is displaced a distance $\mathrm{x}$, we can still write the axial field along the armature element as: 
$B_{z}(\psi)=\frac{\tilde{I}_{i}}{2 \pi \tilde{r}} \frac{\partial}{\partial r} M_{i j}(\tilde{r}), \quad B_{r}(\psi)=-\frac{\tilde{I}_{i}}{2 \pi \tilde{r}} \frac{\partial}{\partial z} M_{i j}(\tilde{r})$

where $\tilde{r}$ and $\psi$ are the radius and angle measured from the unperturbed center (see Fig. (2)), and $\tilde{l}_{i}$ is the perturbed coil current. The net transverse force on the armature element for small displacements can be written as:

$F_{x}=\int_{0}^{2 \pi} d \psi(r \sin \psi) \tilde{I}_{j} B_{z}(\psi)=\frac{1}{2 \pi} \int_{0}^{2 \pi} d \psi \tilde{I}_{i} \tilde{l}_{j} \sin \psi\left(\frac{\partial M_{i j}}{\partial r}+\frac{\partial^{2} M_{i j}}{\partial r^{2}}(\tilde{r}-r)\right)$

Keeping lowest order terms in the displacement we find: $\tilde{r}=r+x \sin (\psi)$ and thus the transverse force to lowest order is given by:

$F_{x}=\frac{l_{i} I_{j}}{2} x \frac{\partial^{2} M_{i j}}{\partial r^{2}}$

In writing Eq. (8) it is assumed that the current density of the armature element under consideration remains uniform and along the azimuthal direction only. This is true for armatures that are thin compared to a skin depth or for litz type elements such as in wound armatures. For a solid projectile, the current flow pattern might become very complex as the projectile is displaced and fields are setup to maintain the magnetic field. To do this problem correctly a 3-D solution is required. However, the above analysis yields an upper limit on the transverse force for the solid projectile case. The reason for this assertion is the fact that for a perfectly conducting projectile, the magnetic field would be frozen implying that the current would also be frozen with respect to unperturbed space as the projectile is displaced. In this case the net transverse force would vanish. For finite resistivity we expect that the net transverse force will be somewhere between the perfectly conducting and uniform current density cases.

In addition to the transverse force, there is a net torque due to the distribution of the axial force in the azimuthal direction, which is given by the third term on the RHS of Eq. (5). Using the expression for the radial magnetic field given by Eq. (6), we find the axial force distribution along the armature element for small displacements is given by: $\quad F_{z}(\psi)=-J_{j} B_{r}(\psi)=\frac{I_{j} J}{2 \pi \tilde{r}}\left(\frac{\partial M_{i j}}{\partial z}+\frac{\partial^{2} M_{i j}}{\partial z \partial r}(\tilde{r}-r)\right)$, where $\mathrm{J}_{\mathrm{j}}$ is the current density of the armature element. This equation assumes that the cross section of the element is small enough so that there is no significant field variation across it. Multiplying by the moment 
arm, with respect to projectile axis $\left(r^{\prime} \sin \left(\varphi^{\prime}\right)\right)$, and integrating over the volume and keeping lowest order terms in displacement we find (see Fig. (2)):

$$
\int d^{3} r^{\prime}\left[r^{\prime} \sin \varphi^{\prime}\right] F_{z}(\psi)=\frac{I_{i} I_{j}}{2} r x \frac{\partial^{2} M_{i j}}{\partial r \partial z}
$$

where $r$ is the armature element radius and $x$ is the displacement. Equations (9) and (10) can now be summed over coil and armature elements and used in the equations of motion,

If the armature element axis is tilted at an angle $\mu$ with respect to the unperturbed axis, we can write the magnetic field due to the coil along the armature element azimuth as:

$$
B_{z}(\psi)=\frac{1}{2 \pi r} I_{i}\left(\frac{\partial M_{i j}}{\partial r}-\frac{\partial^{2} M_{i j}}{\partial r \partial z}(r \mu \sin \psi)\right), \quad \text { and } \quad B_{r}(\psi)=\frac{-1}{2 \pi r} I_{i}\left(\frac{\partial M_{i j}}{\partial z}-\frac{\partial^{2} M_{i j}}{\partial z^{2}}(r \mu \sin \psi)\right)
$$

Above we have assumed that $\mu$ « 1 and kept only lowest order terms. Carrying out the proper integrals over the armature element azimuth, the lowest order transverse force is:

$$
F_{x}=I_{j} \int_{0}^{2 \pi} d \psi r B_{z}(\psi) \sin (\psi)=-\frac{I_{i} I_{j}}{2} \mu r \frac{\partial^{2} M_{i j}}{\partial r \partial z}
$$

The axial force per unit volume along the armature element is $F_{z}(\psi)=-J_{j} B_{r}(\psi)$ and the resulting torque is (third term on RHS of Eq. (5)):

$$
\int d^{3} r^{\prime}\left[r^{\prime} \sin \varphi^{\prime}\right] F_{z}(\psi)=-I_{j} \int_{0}^{2 \pi} d \psi r B_{r}(\psi)[r \sin \psi]=-\frac{I_{i} I_{j}}{2} \mu r^{2} \frac{\partial^{2} M_{i j}}{\partial z^{2}}
$$

Equations (11) and (12) are the required terms in the angular momentum equation. Also note that the sign of the torque is already taken care of in Eq. (5). Another term that is not accounted for in the angular momentum equation is the torque due to the zeroth order radial force, $F_{x}(\psi)=I_{j} B_{z}(\psi) \sin \psi$, combined with a tilt. This torque results from the radial force acting with a moment arm of magnitude $-r \mu \sin \psi$ and is given by:

$$
\tau_{y}=-I_{j} \int_{0}^{2 \pi} d \psi r B_{z}(\psi) \sin \psi[r \mu \sin \psi]=-\frac{I_{i} I_{j}}{2} \mu r_{\frac{\partial}{\partial r}}
$$


Equations (11), (12) and (13) are the force and torque on a tilted armature element due to an unperturbed coil. If the coil is tilted by an angle $\mu$, the projectile-coil system can be rotated by an angle $(-\mu)$ and is thus equivalent to an unperturbed coil and a armature element that is tilted by an angle $(-\mu)$ and displaced by an amount $Z_{i j} \sin (-\mu)$, where $Z_{i j}$ is the axial separation between coil and armature element.

\section{Analysis of Projectile Stability for a Multi-Coil Launcher}

In a real electromagnetic launcher the projectile is made up of many elements, each of which is acted upon by several coils. Since the projectile is assumed rigid, projectile-projectile interactions need not be considered. We assume that each coil has a tilt $\mu_{\mathrm{i}}$ and displacement $\mathrm{x}_{\mathrm{i}}$. Thus the effective tilt for each armature element is $\left(\theta-\mu_{i}\right)$ and the effective displacement is $\left(x_{o}+\theta z_{j}-Z_{i j} \mu_{i}-x_{i}\right)$, where $z_{j}$ is the element's axial position with respect to the projectile center of mass and $\mathrm{Z}_{\mathrm{ij}}$ is the distance between centers of coil and element. Using the above expressions for transverse force and torque due to displacement and tilt and summing over coils and armature elements (replace integrals over $\mathrm{dz}$ ' by summation over armature elements), the equations of motion become:

$M_{p} \frac{d^{2} x_{o}}{d t^{2}}=\sum_{i j} \frac{I_{i} I_{j}}{2} \Lambda_{i j}-2 k l x_{o}(t)$

and

$\frac{M_{p}}{3} l^{2} \frac{d^{2} \theta}{d t^{2}}=\sum_{i j} \frac{I_{i} I_{j}}{2}\left(z_{j} \Lambda_{i j}-2 \theta(t) z_{j \delta^{2}} M_{i j}-\left(\theta-\mu_{i}\right) r_{j \partial r} M_{i j}-\Gamma_{i j}\right)-\frac{2}{3} l^{3} k \theta(t)$

where $\Lambda_{\mathrm{ij}}$ and $\Gamma_{\mathrm{ij}}$ are given by:

$\Lambda_{i j}=\left(\left(x_{o}+z_{j} \theta-x_{i}-Z_{i j} \mu_{i}\right) \frac{\partial^{2} M_{i j}}{\partial r^{2}}-r_{j}\left(\theta-\mu_{i}\right) \frac{\partial^{2} M_{i j}}{\partial r \partial z}\right)$

and

$\Gamma_{i j}=\left(r_{j}\left(x_{o}+z_{j} \theta-x_{i}-Z_{i j} \mu_{i}\right) \frac{\partial^{2} M_{i j}}{\partial r \partial z}-r_{j}^{2}\left(\theta-\mu_{i}\right) \frac{\partial^{2} M_{i j}}{\partial z^{2}}\right)$

The sum over $i$ is over all coils and sum over $j$ is over all armature elements. Equations (14) and (15) can be easily incorporated in the Slingshot code and for a given coil displacement and tilt profiles these equations can be solved to yield the projectile displacement and rotation as a function of time. This can then be used to determine the stresses on the flyway tube. 
Both coil displacement and tilt lead to projectile displacement and rotation of similar magnitudes. Thus without loss of generality we restrict ourselves to the case with only coil displacement. Since the equations of motion need to be solved for $x_{0}$ and $\theta$ as functions of time, it is useful to isolate these variables in Eqs. (14) and (15). Doing this, and assuming all the $\mu_{\mathrm{i}}$ 's are zero, these equations can be written in the form:

$\frac{d^{2} x_{o}}{d t^{2}}=A x_{o}+B \theta+C$

$\frac{d^{2} \theta}{d t^{2}}=\alpha x_{o}+\beta \theta+\gamma$

where the coefficients are functions of time and are given by:

$$
\begin{aligned}
& A=\frac{1}{M_{p}}\left[\left(\sum_{i j} \frac{I_{i} I_{j} \partial^{2} M_{i j}}{\partial r^{2}}\right)-2 k l\right] \quad B=\frac{1}{M_{p}} \sum_{i j}\left(\frac{I_{i} I_{j}}{2}\right)\left(z_{j} \frac{\partial^{2} M_{i j}}{\partial r^{2}}-r_{j} \frac{\partial^{2} M_{i j}}{\partial r \partial z}\right) \\
& C=\frac{-1}{M_{p}} \sum_{i j} \frac{I_{i} I_{j}}{2} x_{i} \frac{\partial^{2} M_{i j}}{\partial r^{2}} \quad \alpha=3 \frac{B}{l^{2}} \\
& \beta=\frac{3}{l^{2} M_{p}}\left[\sum_{i j}\left(\frac{I_{i} I_{j}}{2}\right)\left(z_{j}^{2} \frac{\partial^{2} M_{i j}}{\partial r^{2}}-2 z_{j} r_{j} \frac{\partial^{2} M_{i j}}{\partial r \partial z}+r_{j}^{2} \frac{\partial^{2} M_{i j}}{\partial z^{2}}-2 z_{j} \frac{\partial M_{i j}}{\partial z}-r_{j} \frac{\partial M_{i j}}{\partial r}\right)-\frac{2}{3} k l^{3}\right] \\
& \gamma=-\frac{3}{l^{2} M_{p}} \sum_{i j}\left(\frac{I_{i} I_{j}}{2}\right)\left(z_{j} \frac{\partial^{2} M_{i j}}{\partial r^{2}}-r_{j} \frac{\partial^{2} M_{i j}}{\partial r \partial z}\right) x_{i}
\end{aligned}
$$

The terms $C$ and $\gamma$ are the forcing terms and they are proportional to the displacement of the coils. Since we only considered first order terms in displacement and rotation the equations of motion are linear and thus are only valid for small perturbations. Even though all the terms in the coefficients of Eqs. (16) and (17) have strong time dependence, we expect that these coefficients represented by the sums over coils and armature elements are only slowly varying in time similar to the axial acceleration. Because of the slow time variation (over many coils) we can approximate these coefficients by constants and get a reasonably good idea about the resonant frequencies of the launcher. These resonant frequencies are found from the roots of the characteristic equation which is obtained by assuming solutions of the form $e^{i \omega t}$ in the homogeneous equations of motion, that is roots of the equations:

$$
-\omega^{2} x_{o}-A x_{o}-B \theta=0 \text { and }-\omega^{2} \theta-\alpha x_{o}-\beta \theta=0
$$


with solutions:

$$
\omega^{2}=\frac{-(A+\beta) \pm \sqrt{(A-\beta)^{2}+4 \alpha B}}{2}
$$

Equation (18) shows that there is the possibility of unstable solutions, depending on the values of the coefficients. If $A$ and $\beta$ are negative and $|A+\beta|>\sqrt{(A-\beta)^{2}+4 \alpha B}$ then all solutions are stable, otherwise projectile transverse motion is unstable. We note that for all values of the coefficients, there exists a value of the flyway restoring force constant $k$ that would make $A+\beta$ large enough negative to stabilize the motion.

For a stable projectile, there are two frequencies given by Eq. (18) at which the projectile can naturally oscillate. If the coils are displaced at a certain axial wavelength $\lambda$, the forcing terms given by $\mathrm{C}$ and $\gamma$ in Eqs. $(16,17)$ will have a time dependence roughly of the form $e^{i \omega_{o} t}$, where $\omega_{o}=\left(2 \pi v_{z}\right) / \lambda$, and $v_{z}$ is the projectile axial speed. If $\omega_{0}$ matches one of the natural frequencies, a resonance condition will develop which will result in a secularly growing oscillation amplitude. However, since $v_{z}$ is a changing function of time, this resonance condition will not be maintained. After the resonance, the projectile will continue to oscillate at its natural frequencies with a contribution due to the forcing term. Physically it is expected that coil displacement will be randomly distributed axially in the launcher. In such a case growth of the displacement amplitude due to resonance will depend on the distribution of wavelengths in the displacement profiles.

\section{Numerical Solution and Results}

As mentioned previously, the complexity of the coefficients of the equations of motion necessitates a numerical solution in order to obtain quantitative information about stability and displacement growth. Implementation of such a numerical solution is straightforward in the Slingshot code. Slingshot is a 2-D simulation code that represents electromagnetic interactions between coils and armature elements by a circuit model. The circuit equations solved are very similar to those in the WARP-10 code described in Ref. (3). The equations of motion are then integrated using the normal Slingshot time step in a leap-frog algorithm.

Several simulations were run for a 20 meter launcher with a solid aluminum armature. The parameters used are as follows: Projectile: $\mathrm{IR}=0.055 \mathrm{~m}, \mathrm{OR}=0.095 \mathrm{~m}$, Length $=2 l=0.4 \mathrm{~m}$ and the total accelerated mass is $60 \mathrm{~kg}$. Coils: (270 stages) $\mathrm{IR}=0.105 \mathrm{~m}, \mathrm{OR}=0.185 \mathrm{~m}, \Delta z=0.06 \mathrm{~m}$, center to center spacing $=$ $0.075 \mathrm{~m}$ and fill factor $=0.2$. The circuit and parameters used is shown in Fig. (3). The voltage for the first 30 stages is graded nearly linearly to a maximum value of $40 \mathrm{kV}$ in order to reduce armature temperature rise. The number of turns in the coils is determined by the code based on a constant rise length of $18 \mathrm{~cm}$ and 
a slip distance proportional to the projectile position is used (with proportionality constant of $12 \times 10^{-3}$ ). For these parameters the final velocity is $V_{f}=1791 \mathrm{~m} / \mathrm{sec}$. For the above parameters, the value of the flyway spring constant for which the displacement solution is stable is $k \approx 1.0 \times 10^{8} \mathrm{~N} / \mathrm{m}^{2}$. This is also the value for which $\omega_{2}^{2}(t)$ is non-negative for all time.

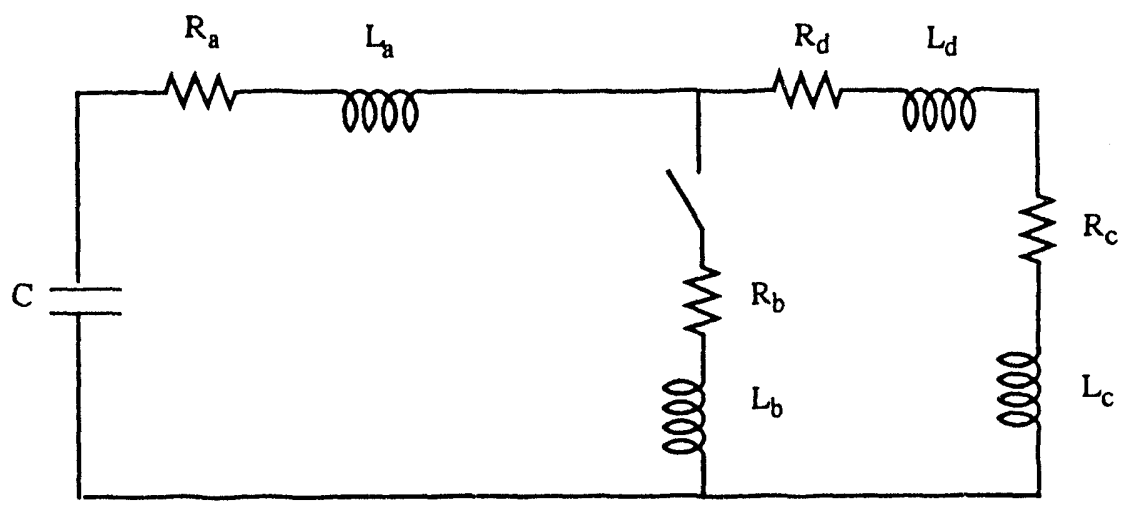

Fig. 3. Circuit diagram for all stages. $R_{a}=1 \mathrm{~m} \Omega, R_{b}=25 \mathrm{~m} \Omega, R_{d}=1 \mathrm{~m} \Omega$. $C=1600 \mu \mathrm{F}$. $\mathrm{L}_{\mathrm{a}}=0.1 \mu \mathrm{H}, \mathrm{L}_{\mathrm{b}}=2.5 \mu \mathrm{H}, \mathrm{L}_{\mathrm{d}}=0.1 \mu \mathrm{H}$.

We performed simulations with $k=1.5 \times 10^{8} \mathrm{~N} / \mathrm{m}^{2}$ and using the following coil displacement profile: $x_{i}=A_{o} \sin \left(\frac{2 \pi z_{i}}{\lambda}\right)$, where $z_{i}$ and $x_{i}$ are the axial location and displacement of the $i$ th coil respectively and $A_{o}$ and $\lambda$ are the imposed amplitude and wavelength. In Fig. (4) the projectile axial history, the resulting natural frequencies $\omega_{1}$ and $\omega_{2}$ and the displacment and rotation are shown for $A_{0}=1 \mathrm{~mm}$ and $\lambda=1$ meter. Although the coefficients of the equations of motion vary with time (as seen in the dependence of $\omega_{1}$ and $\omega_{2}$ ), the basic mechanism for growth of the transverse displacement and rotation is as discussed in the previous section, namely due to resonant interaction with the driving forces. The maximum oscillation amplitude is about $4 \mathrm{~mm}$ for this simulation and thus the transverse force per unit length exerted by the flyway tube is: $F_{r}=k x_{o}=6 \times 10^{5} \mathrm{~N} / \mathrm{m}$. If this force is distributed over a fraction $\mu$ of the circumference, the pressure is: $P=F_{r} /(2 \pi R \mu)$, which amounts to 50 bars for $\mu=20 \%$. For a more accurate estimate of the transverse pressure, the restoring force law needs to be modified to account for the detailed structures used in the launcher. The projectile transverse velocity at the muzzle is roughly $5 \mathrm{~m} / \mathrm{sec}$, which is less than $0.3 \%$ of the axial velocity. The rotation frequency at the muzzle $(\mathrm{d} \theta / \mathrm{dt})$ is roughly $3 \mathrm{~Hz}$, which can be easily spin stabilized.

Results of a simulation for a wound armature for similar parameters are shown in Fig. (5). This armature has a fill factor of 0.8 and is modeled by assuming uniform current density throughout. Also, the coil voltage is reversed at stage 150 to improve the efficiency. Transverse motion results are very similar to the solid armature case. 
Simulations with random coil displacement also show similar growth in amplitudes. The reason for this growth is the existence of long wavelengths components in the driving forces. A general coil displacement profile can be analyzed using Fourier decomposition.

In the figures, the transverse oscillations continue after the projectile leaves the launcher ( 20 meters). This is because the flyway restoring forces are not turned off in the simulations after the projectile leaves. After the projectile leaves, all the coil forces go to zero and the limit of the natural frequency becomes: $\omega_{1}=\omega_{2}=\sqrt{2 k l / M_{p}}$ which is the observed frequency. The amplitude of these oscillations depends on the phase of the oscillation when the projectile exits the launcher.

\section{Summary of Results}

The equations of motion for both translation and rotation of a projectile in an electromagnetic induction launcher were derived. The electromagnetic interactions between coils and displaced or tilted armature elements were calculated based on a first order perturbation method, assuming that the perturbed current flows in an azimuthally symmetric fashion with respect to the displaced armature element. To account for the breaking of this symmetry a 3-D model is required in which fields and currents would have to be calculated at all points in the projectile. The usefulness of the present model is the fact that it provides an upper limit estimate of the transverse forces and displacements and the relative ease of incorporating it in the 2-D Slingshot code.

Depending on the parameters, the transverse motion cain be unstable. However, by increasing the spring constant for the flyway tube the motion can be stabilized in those situations. For the 20 meter launcher parameters, and restoring force per unit length proportional to the local displacement, the value of the spring constant for which stable motion occurs is $k \approx 1.0 \times 10^{8} \mathrm{~N} / \mathrm{m}^{2}$. Numerical simulations were performed for $k=1.5 \times 10^{8} \mathrm{~N} / \mathrm{m}^{2}$ for both solid and wound armatures and the resulting displacements are of the order of $4 \mathrm{~mm}$, for coil displacements of $1 \mathrm{~mm}$ amplitude. For these values, a rough estimate of the transverse pressure on the flyway tube is about 50 bars. The growth in amplitude is due to a resonant interaction between projectile and the coil displacement profile. This resonant interaction does not persist because of the rapidly changing axial velocity. The higher the axial velocity, the longer the wavelength required for resonance. The restoring force law used in the above analysis is highly idealized and is certainly not adequate to describe the complex structure of a launcher. An accurate description of the restoring force law is needed in which both axial and transverse dependences are prescribed. Such a prescription can be easily implemented in the numerical solution. 

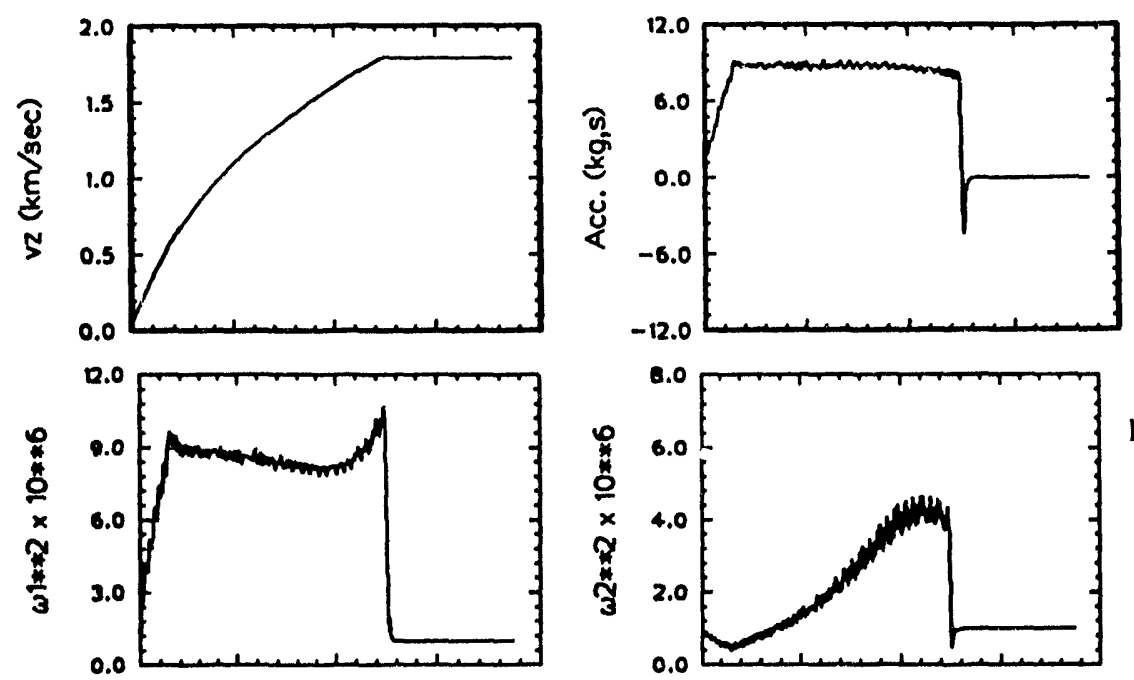

Fig. 4. Results of Slingshot simulation for a solid armature. Transverse motion for coil displacement of the form: $x_{i}=A_{o} \sin \left[\left(2 \pi z_{i}\right) / \lambda\right]$, for $A_{0}=1 \mathrm{~mm}$ and $\lambda=1$ meter
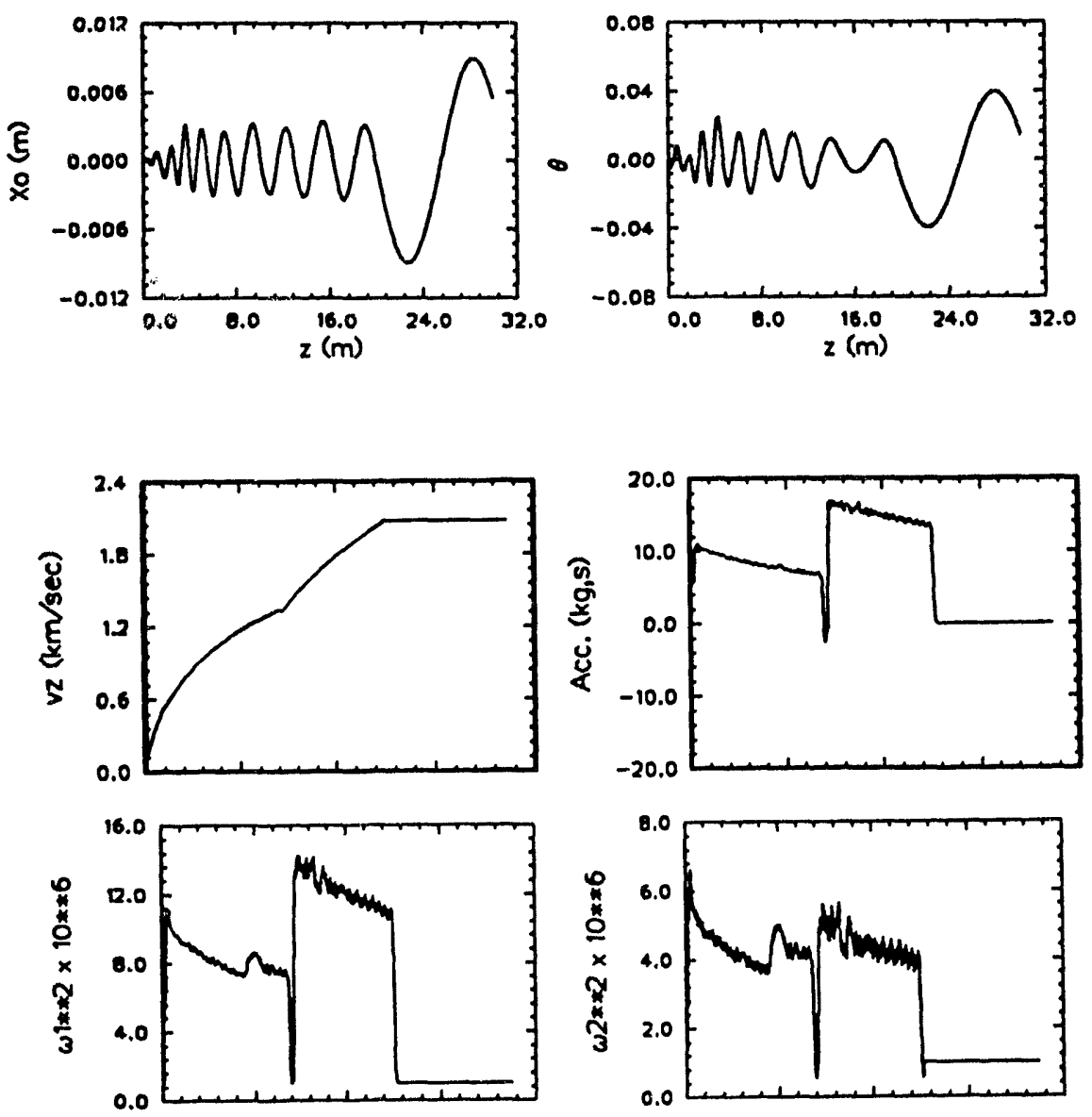

Fig. 5. Results of Slingshot simulation for a wound armature. Coil voltage is reversed at stage 150 . The rise length is $18 \mathrm{~cm}$ and the firing positions are advanced for the stages by: $100 @ 16 \mathrm{~cm}$, 50@18,3@-6,117@12 cm. This advance in firing position results in optimized performance. Same displacment profile as Fig. 4.
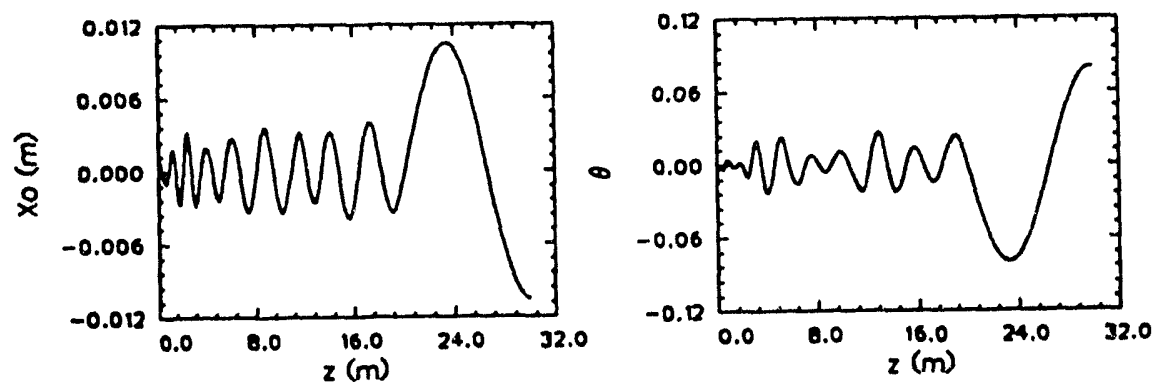


\section{References}

1- M. Cowan, M. M. Widner, E. C. Cnare, B. W. Duggin, R. J. Kaye and J. R. Freeman, "Exploratory Development of the Reconnection Launcher 1986-1990", IEEE Transactions on Magnetics, Vol. 27, No. 1, January 1991.

2- R. J. Kaye, E. L. Brawley, B. W. Duggin, E. C. Cnare, D. C. Rovang and M. W. Widner, "Design and Performance of a Multi-Stage Cylindrical Reconnection Launcher", IEEE Transactions on Magnetics, Vol. 27, No. 1, January 1991.

3- M. M. Widner, "WARP-10: A Numerical Simulation Model for the Cylindrical Reconnection launcher", IEEE Transactions on Magnetics, Vol. 27, No. 1, January 1991.

4- M. Cowan and I. R. Shokair, “Advantages of a Wound Armature”, Sandia Internal memo, March, 1993.

5- B. M. Marder, Private Communication.

6- I. R. Shokair and M. Cowan, "Summary of Navy Gun Simulations", Sandia Internal memo April 20, 1993.

\section{Acknowledgments}

The author would like to thank M. Cowan, R. Kaye and B. Marder for useful discussions and comments. This work was supported by the Department of Energy under Contract No. DE-AC04-76DP00789

\section{DISCLAIMER}

This report was prepared as an account of work sponsored by an agency of the United States Government. Neither the United States Government nor any agency thereof, nor any of their employees, makes any warranty, express or implied, or assumes any legal liability or responsibility for the accuracy, completeness, or usefulness of any information, apparatus, product, or process disclosed, or represents that its use would not infringe privately owned rights. Reference herein to any specific commercial product, process, or service by trade name, trademark, manufacturer, or otherwise does not necessarily constitute or imply its endorsement, recommendation, or favoring by the United States Government or any agency thereof. The views and opinions of authors expressed herein do not necessarily state or reflect those of the United States Government or any agency thereof. 


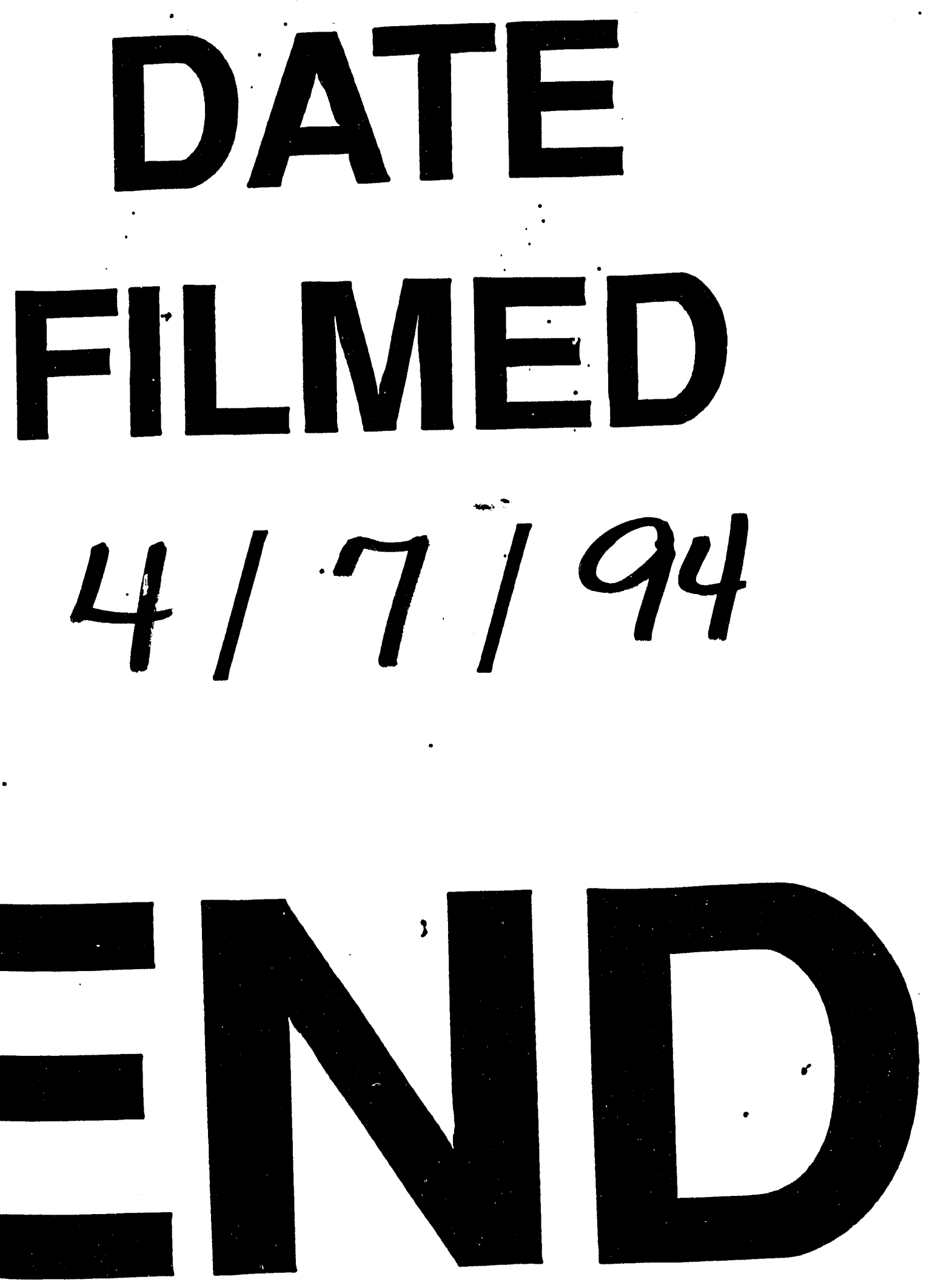


